\title{
MULTILAYER ALD CERAMIC FILMS FOR ENHANCEMENT OF PARYLENE BARRIER PROPERTIES IN COMPLIANT NEURAL PROBES WITH BONDED CHIPS
}

\author{
Mats Forssell, Xiao Chuan Ong, and Gary K. Fedder*
}

Carnegie Mellon University, Pittsburgh, Pennsylvania, USA

\begin{abstract}
The insulation capabilities of parylene-C thin films in phosphate buffered saline (PBS) are improved through their combination with $\mathrm{ALD}$ nanolaminate $\mathrm{Al}_{2} \mathrm{O}_{3}$ and $\mathrm{TiO}_{2}$ films. Insulation structures sealed with this ceramic exhibit a $5 \mathrm{X}$ lower failure rate compared to those sealed with parylene alone. The insulation of parylene-C probes including $50 \mu \mathrm{m}$-thick $\mathrm{Si}$ chips bonded using anisotropic conductive film (ACF) is also investigated. The insulation is less effective with the bonded chip present, but samples maintain an impedance above $1 \mathrm{G} \Omega$ at $0.1 \mathrm{~Hz}$ for over 5 weeks. Addition of nanolaminate results in a lower, but more stable impedance.
\end{abstract}

\section{INTRODUCTION}

Despite its excellent mechanical properties, parylene-C has not been conclusively demonstrated as a viable electrical insulating material for long-term biological implantation, as would be desirable for its use in compliant neural sensors. This is in part because its insulating characteristics degrade over a period of hours to days when inserted in body fluid[1]-[7]. Because ceramic materials have a lower water vapor transmission rate (WVTR), they are a promising alternative for long-term in vivo insulation. Atomic layer deposition (ALD) allows deposition of sub-nanometer thick layers of ceramic oxides. Therefore compliant probes can be made in spite of the higher stiffness of the materials, using thin $(<100 \mathrm{~nm})$ layers.

Multilayer insulations combining parylene and ceramic have ultimately proven ineffective using $\mathrm{Al}_{2} \mathrm{O}_{3}$ [8]. However, nanolaminates formed by alternating monolayers of $\mathrm{Al}_{2} \mathrm{O}_{3}$ and $\mathrm{TiO}_{2}$ have shown superior water corrosion resistance[9], [10]. Silicon carbide thin films have also been used to insulated neural probes[11], and have shown good compatibility with polyimide[12]. However, combinations of $\mathrm{Al}_{2} \mathrm{O}_{3}$ and $\mathrm{TiO}_{2}$ provide greater corrosion resistance than thin films of $\mathrm{SiC}$ in hot saline[13] Combining parylene and $\mathrm{Al}_{2} \mathrm{O}_{3} / \mathrm{TiO}_{2}$ nanolaminate is a promising avenue for extending the lifetime of parylene neural probes.

Highly reliable insulation is especially critical for the development of active compliant parylene probes. Active chips can be used to increase the electrode count on neural probes, and increase the recorded signal quality. However, the DC voltage required to power the probe accelerates the degradation of the insulation. Moreover, failure of the insulation can result in not only the expected probe failure, but also cause high leakage currents in the tissue, which can be hazardous. $1.8 \mathrm{~V}$ is a commonly used supply voltage for analog CMOS designs due to an excellent combination of performance and cost. Even operated on a dual rail $\pm 0.9 \mathrm{~V}$, the voltage required exceeds the electrochemical water window of [-0.6 V; $0.8 \mathrm{~V}$ ] (against $\mathrm{Ag} / \mathrm{AgCl}$ )[14].

The addition of chips in parylene thin films [15] was previously shown to degrade the insulation capabilities due to cracking of the insulation when tested at $77^{\circ} \mathrm{C}$ in saline. A more reliable integration method that maintains a high insulation quality is therefore required.

\section{FABRICATION}

\section{Insulation structures}

Test structures are fabricated to characterize the insulation properties of parylene with additional nanolaminate films. First, a 4"-diameter glass wafer (Soda Lime, University Wafer, Boston, MA) is dipped in a solution of A-174 adhesion promoter (A174:H2O:IPA $=1: 100: 100$ by volume) and air dried. A $2 \mu \mathrm{m}$-thick layer of parylene-C is deposited on the wafer (SCS Labcoter 2, Specialty Coating Systems, Inc., Indianapolis, IN) (Fig. 1(a)). Atomic layer deposition (ALD) is used to deposit $6 \mathrm{~nm}$ of $\mathrm{TiO}_{2}$ as an adhesion layer, followed by $80 \mathrm{~nm}$ of $\mathrm{Al}_{2} \mathrm{O}_{3} / \mathrm{TiO}_{2}$ nanolaminate (Fiji Cambridge Nanotech system, Veeco Instruments, Inc., Plainview, NY) formed by alternating the deposition of $\mathrm{Al}_{2} \mathrm{O}_{3}$ and $\mathrm{TiO}_{2}$ layers (Fig. 1(b)). A $125 \mathrm{~nm}$ Pt layer is sputter deposited (6J, Perkin-Elmer) with $10 \mathrm{~nm}$ Ti adhesion layer on both sides and patterned via liftoff (Fig. 1(c)). A second $80 \mathrm{~nm}$ layer of nanolaminate is deposited, followed by a $6 \mathrm{~nm}$ layer of $\mathrm{TiO}_{2}$ adhesion layer (Fig. 1(d)). The nanolaminate is etched in $\mathrm{Cl}_{2}$ inductively coupled plasma reactive ion etch (Versaline ICP-RIE system, Plasma-Therm, St. Petersburg, FL) (Fig. 1(e)). A wet etch using buffered hydrofluoric acid (BHF) was also investigated, but residue led to a poor insulation quality (Fig. 2). A final $2 \mu \mathrm{m}$ parylene layer is deposited, following which the parylene-C is etched using $\mathrm{O}_{2}$ RIE (Trion Technology, Inc., Tempe, AZ) using a $\mathrm{Cr}$ hard mask. Individual dies are heated for $3 \mathrm{~h}$ at $300{ }^{\circ} \mathrm{C}$ in a vacuum chamber at 10 mTorr using $\mathrm{N}_{2}$ backfill to anneal the parylene layers, which improves the insulation [6].

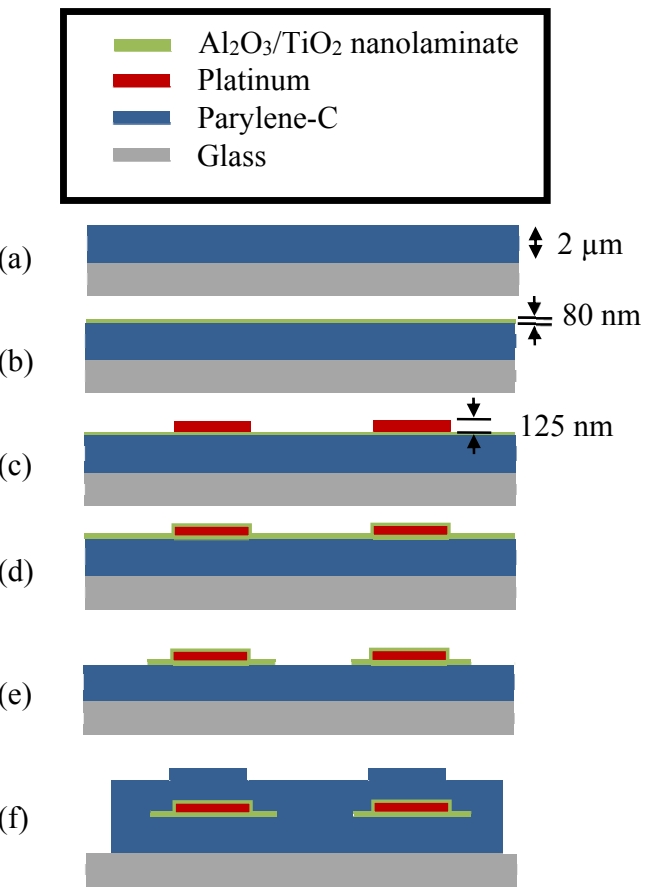

Figure 1: Insulation test structure fabrication simplified process flow 


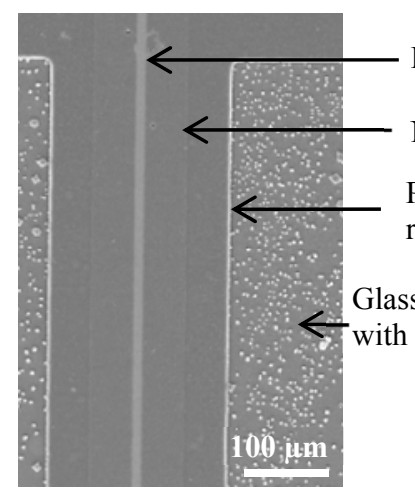

(a)

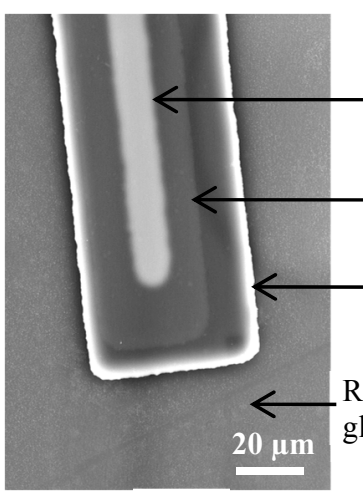

(c)

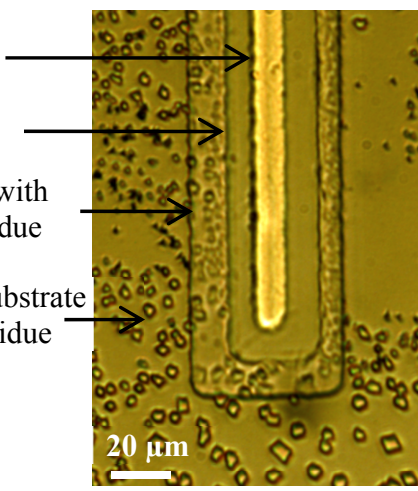

(b)

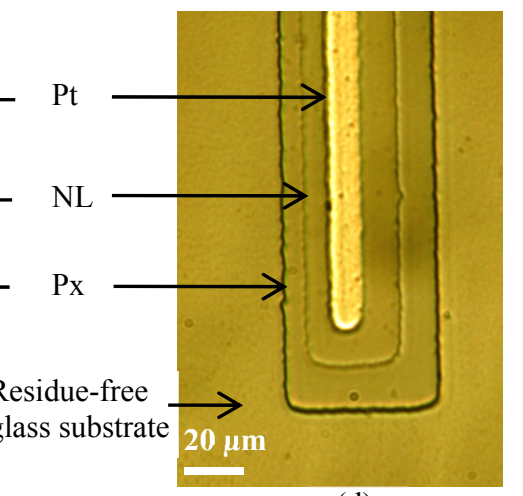

(d)
Figure 2: SEM (a),(c) and optical (b),(d) images of insulated Pt wires in which the NL layer was etched using BHF (a),(b) and RIE (c),(d)

Each fabricated sample contains 16 metal traces that are $10 \mu \mathrm{m}$ wide and $2 \mathrm{~cm}$ long. The ceramic insulation surrounding these wires varies from $10 \mu \mathrm{m}$ to $100 \mu \mathrm{m}$ in width, while a continuous parylene layer covers all the wires. Samples without ceramic encapsulation were also fabricated. The samples are inserted in phosphate buffered saline (PBS), with around $1 \mathrm{~cm}$ of the insulated traces immersed, and placed in a sandbath at either $37^{\circ} \mathrm{C}$ or $60^{\circ} \mathrm{C}$. Periodically, the samples are removed from the sandbath and the impedance between the insulated traces and a counter electrode is measured at room temperature using a potentiostat (PGSTAT301N, Metrohm, AG, Herisau, Switzerland).

\section{ACF chip bonding}

In the chip bonding experiments, $1 \mathrm{~mm}$ by $1.2 \mathrm{~mm}$ by $50 \mu \mathrm{m}-$ thick silicon chips are integrated in parylene thin films using anisotropic conductive film (CP34531-18AB, Dexerials Corporation, Tokyo, Japan). The chips are bonded before the final parylene deposition (Fig. 1(f)) using a flip-chip die bonder (M9A, BE Semiconductor Industries N.V. (Besi), Duiven, The Netherlands). For bonding tests, the dimensions of the die is $1.2 \mathrm{~cm}$ by $0.9 \mathrm{~cm}$, and the parylene thickness is $4.5 \mu \mathrm{m}$ for each layer. Si is used as a substrate for parylene/ceramic for simpler processing.

Bonding conditions are optimized to ensure that electrical connection between the chip and the substrate is made, without damage to the underlying films. Excessive bonding pressure causes breakage of the platinum wiring, resulting in loss of electrical connection (Fig. 3). Cracks in the platinum could also propagate through the insulation stack and result in leakage.

The presence of protruding pads on the chips facilitates
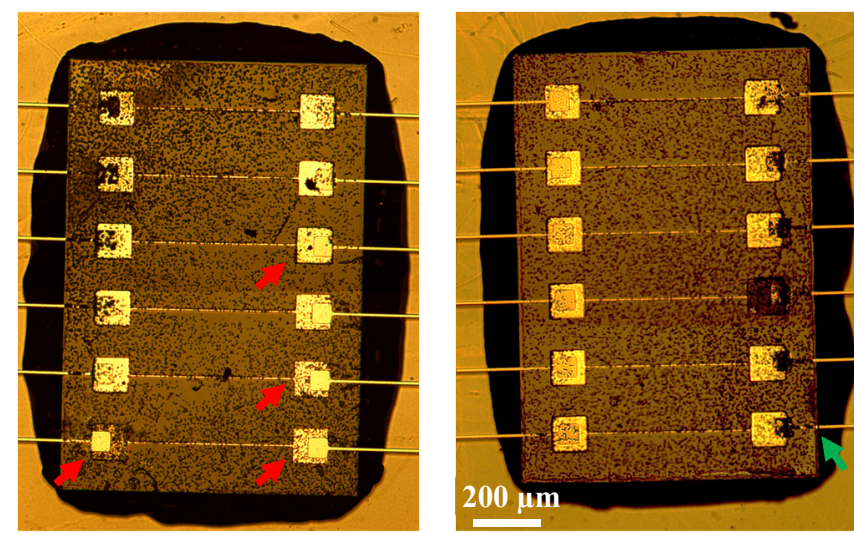

Figure 3: Bottom view of chips without protruding pads connected to probes via $A C F$. The black dots are the ACF conductive microparticles. The lack of balls on pads indicated by red arrows indicate that the electrical contact is not made. A break in the wire at the edge of the chip is indicated by a green arrow. The left chip was bonded with $4 \mathrm{~kg}$ of force, the right chip with $8 \mathrm{~kg}$.

bonding by reducing the requirement on planarity: without protruding pads, a slight tilt between the chip and probe results in a high force applied at the edge of the chip, as seen in Fig. 3. A $2.2 \mu \mathrm{m}$ protrusion is sufficient to ensure that the electrical connection is made with high yield.

\section{RESULTS AND DISCUSSION}

\section{Insulation testing}

The impedance at $0.1 \mathrm{~Hz}$ in PBS provides a metric for the quality of the insulation. For non-defective insulated samples, the $0.1 \mathrm{~Hz}$ impedance theoretically follows an exponential decay

$$
|Z|=Z_{0} \exp (-t / \tau)
$$

However, defects in the insulation result in some channels having a more rapid impedance drop. Empirically, a minimum limit criterion of $Z_{L}=\left(10^{10} \Omega\right) \exp (-t / 2000 \mathrm{~h})$ allows to distinguish between nondefective and defective channels. Figure 4 shows the impedance at $37^{\circ} \mathrm{C}$ averaged over 16 channels for non-defective samples, as well as the proportion of non-defective channels over time.
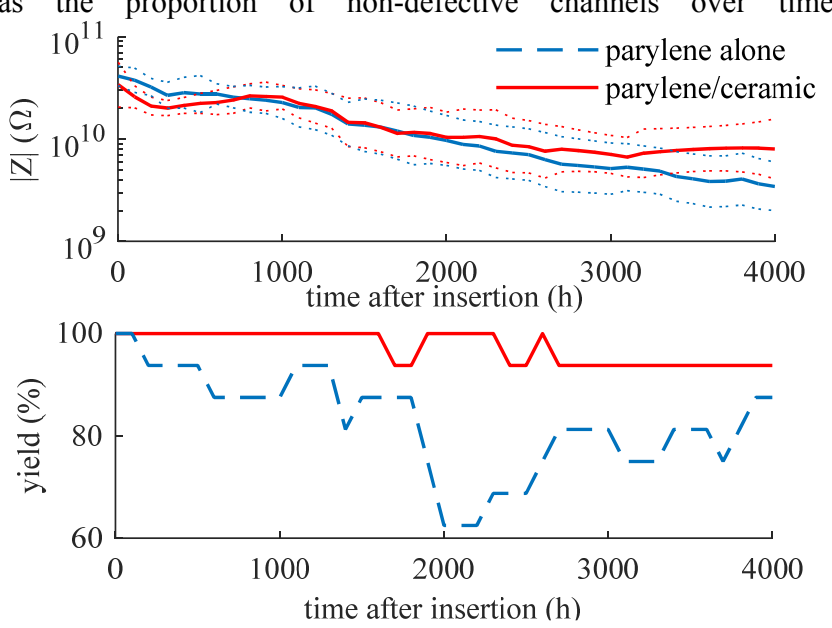

Figure 4: Comparison of (top) $0.1 \mathrm{~Hz}$ impedance variation at $37^{\circ} \mathrm{C}$ and (bottom) proportion of functional channels ( $n=16$ channels). The dotted lines in the impedance plot denote one standard deviation from the mean (solid line).

The time constant for the impedance decay is extracted by fitting the impedance to (1) and the failure rate is extracted by fitting the yield 
to a linear decrease (Table 1). Because the impedance decay is initially faster, the fitting is limited to $t>1000 \mathrm{~h}$. The parylene/ceramic insulation has a lower impedance decrease for non-defective channels, consistent with the improvement in insulation expected from using the additional ceramic layer. In addition, the samples with ceramic have a lower number of defective channels. This indicates that the additional ALD insulation is less susceptible to defects during fabrication.

Table 1: Performance comparison at $37^{\circ} \mathrm{C}$

\begin{tabular}{|l|c|c|}
\cline { 2 - 3 } \multicolumn{1}{c|}{} & $\begin{array}{c}\text { parylene-C } \\
\text { alone }\end{array}$ & $\begin{array}{c}\text { parylene-C/ } \\
\text { ceramic }\end{array}$ \\
\hline Time constant $(\mathrm{h})$ & 1639 & 3253 \\
\hline Failure rate $(\% / \mathrm{h})$ & 0.76 & 0.16 \\
\hline
\end{tabular}

Using a higher temperature of $60^{\circ} \mathrm{C}$ accelerates the degradation of the insulation. The samples insulated using parylene alone experience a rapid decrease in impedance of over 10 times in a short period of time $(<24 \mathrm{~h})$. This rapid decrease associated with a total insulation failure only occurs after $500 \mathrm{~h}$ with the ceramic.

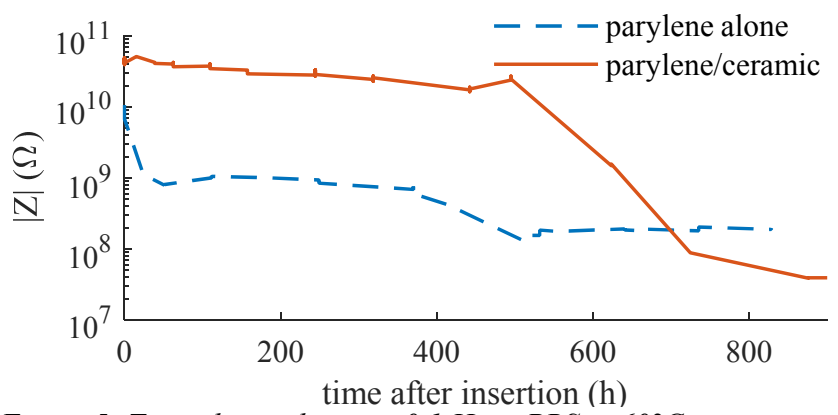

Figure 5: Typical impedance at $0.1 \mathrm{~Hz}$ in $\mathrm{PBS}$ at $60^{\circ} \mathrm{C}$.

\section{Bonded chip insulation}

Bonding test structures consist of insulated partial loops as shown in Fig. 6. When the chip is electrically connected, the loop resistance is between 1-2 $\mathrm{k} \Omega$; most of the resistance originates from the platinum film resistance. If the bond fails due to a poor ACF connection, or because of cracking of the platinum film, the loop becomes an open circuit.

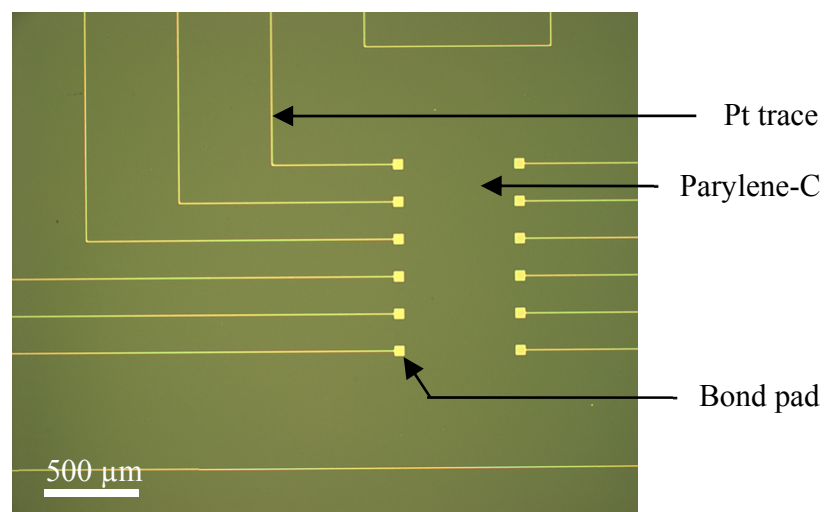

Figure 6: Photograph of a test structure prior to bonding. The bonded chip forms a connection between the right and left pads

Fig. 7 shows the impedance at $0.1 \mathrm{~Hz}$ of surviving loops over $900 \mathrm{~h}$ of insertion in PBS at room temperature. After $300 \mathrm{~h}$, a $3 \mathrm{~V}_{\mathrm{pp}}$ sinusoidal voltage with frequency $33 \mathrm{mHz}$ was applied continuously to the loops. After $500 \mathrm{~h}$, the voltage stimulation was changed to a
$3 \mathrm{~V}_{\mathrm{pp}}$ square wave with a frequency of $10 \mathrm{mHz}$. The additional voltage stimulation does not appear to change the insulation characteristics over short time scales.

After chip bonding under a $1 \mathrm{~kg}$ load, 4/6 loops survived for the parylene alone case, while only $1 / 6$ loops survived for parylene/ceramic insulation. Most of the loop failures occurred during fabrication, with only one failure occurring after PBS insertion, in a sample without ceramic. Because the nanolaminate is more brittle than parylene, its presence increases the likelihood of cracks during the chip bonding and the subsequent processing steps. Improvements in that assembly step are ongoing to improve the fabrication yield.

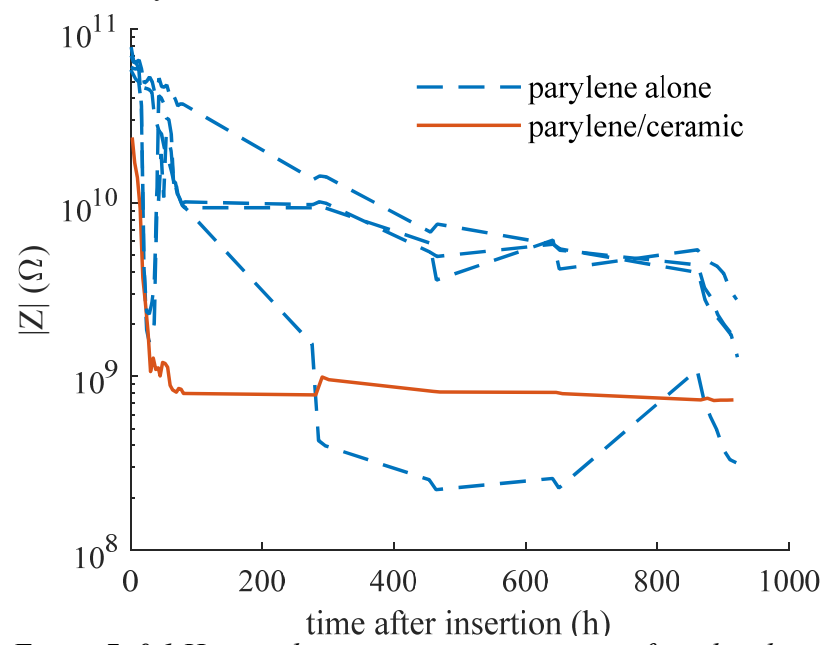

Figure 7: $0.1 \mathrm{~Hz}$ impedance at room temperature of insulated traces including a bonded chip.

The time constants obtained from fitting to (1) are $356 \mathrm{~h}, 570 \mathrm{~h}$, $626 \mathrm{~h}$, and $837 \mathrm{~h}$ for the four parylene-alone samples. The time constant measured without the bonded chip (Table 1) is between 2 and 4 times larger. This is partly because the impedance decreases faster shortly after insertion in PBS, but the presence of the chip also results in a poorer insulation.

In the case of parylene with nanolaminate, the impedance decreases quickly, but stabilizes around $1 \mathrm{G} \Omega$. Even though the parylene insulation fails, the nanolaminate layer provides a barrier to prevent additional leakage. Compared to samples with parylene alone, the $1 \mathrm{G} \Omega$ impedance at $0.1 \mathrm{~Hz}$ mainly results from the thin nanolaminate dielectric layer, which displays a more capacitive characteristic. The phase is around $-15^{\circ}$ for parylene alone and around $-45^{\circ}$ for parylene/ceramic

Because active chips must be powered with voltages outside the electrochemical water window, the current will increase exponentially with voltage. Therefore relying on the small-signal impedance spectroscopy measurement does not guarantee that high currents might not happen at higher voltages. Cyclic voltammetry (CV) performed between $-1.5 \mathrm{~V}$ and $+1.5 \mathrm{~V}$ at a rate of $0.1 \mathrm{~V} / \mathrm{s}$ verifies that nonlinear increases in current do not occur in the normal range of operation of CMOS chips. Fig. 8 shows that leakage currents for both parylene and parylene/ceramic are limited to 1.5 $\mathrm{nA}$ within the $-0.9 \mathrm{~V}$ to $+0.9 \mathrm{~V}$ supply range. The capacitive nature of the parylene/ceramic sample is apparent in the gap between the current measured when the applied potential is increasing compared to when the potential is decreasing. 


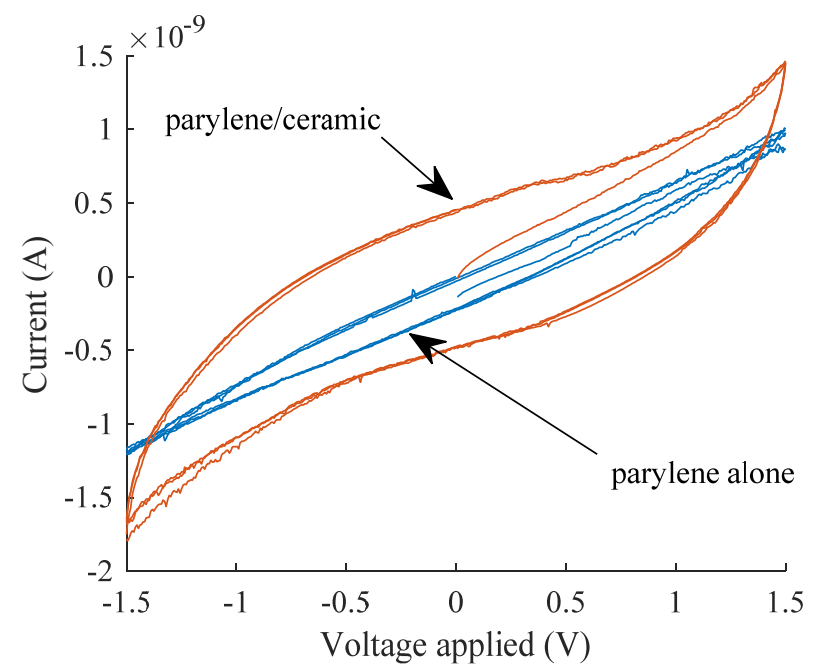

Figure 8: Cyclic voltammetry performed after $900 \mathrm{~h}$ in PBS on insulated traces including a bonded chip.

\section{CONCLUSION}

The insulation of parylene thin film structures on glass is improved by the addition of an ALD ceramic $\mathrm{Al}_{2} \mathrm{O}_{3} / \mathrm{TiO}_{2}$ nanolaminate. The nanolaminate reduces the susceptibility of the samples to defects, enabling a five-fold reduction in failure rate. Additionally, it provides an additional barrier to leakage, resulting in a two-fold improvement in impedance decrease.

The high-performing insulation allows the development of probes containing active circuits. A simple process for integrating chips in parylene structures using ACF is demonstrated. The insulation performance is degraded when the chip is added, but leakage currents stay below $1.5 \mathrm{nA}$ with $\pm 0.9 \mathrm{~V}$ operation for over $900 \mathrm{~h}$. The nanolaminate layer results in an impedance that is initially lower, but is more stable over long time periods. The results demonstrate that active chips embedded into parylene thin films can reliably operate for over a month.

Improvements in the fabrication process to increase the yield are underway: cracking in the insulation layers is thought to be caused by compliance mismatch between the rigid chip and the flexible layers; a gradual change in transition between the two regions can lower the stress at the interface. These measurements were performed on glass substrates; since compliant probes is a main application of parylene, measurement of insulation of released parylene structures is a logical next step.

\section{ACKNOWLEDGEMENT}

We thank Dr. Matt Moneck and his staff at the Carnegie Mellon Nanofabrication Facility for equipment and process support. Part of this work was sponsored by the Defense Advanced Research Projects Agency (DARPA) ElectRx Program under the auspices of Dr. Doug Weber through the Space and Naval Warfare Systems Center Contract No. HR0011-15-2-0009.

\section{REFERENCES}

[1] H. Yasuda, Q. . Yu, and M. Chen, "Interfacial factors in corrosion protection: an EIS study of model systems," Prog. Org. Coatings, vol. 41, no. 4, pp. 273-279, May 2001.

[2] W. Li, D. Rodger, P. Menon, and Y. Tai, "Corrosion behavior of parylene-metal-parylene thin films in saline," ECS Trans., vol. 11, no. 18, pp. 1-6, 2008.
[10] L. H. Kim, K. Kim, S. Park, Y. J. Jeong, H. Kim, D. S. Chung, S. H. Kim, and C. E. Park, "Al 2 O $3 /$ TiO 2 Nanolaminate Thin Film Encapsulation for Organic Thin Film Transistors via Plasma-Enhanced Atomic Layer Deposition," ACS Appl. Mater. Interfaces, vol. 6, no. 9, pp. 6731-6738, May 2014.

[11] X. Lei, S. Kane, S. Cogan, H. Lorach, L. Galambos, P. Huie, K. Mathieson, T. Kamins, J. Harris, and D. Palanker, "SiC protective coating for photovoltaic retinal prosthesis," J. Neural Eng., vol. 13, no. 4, p. 46016, 2016.

[12] J. S. Ordonez, C. Boehler, M. Schuettler, and T. Stieglitz, "Improved polyimide thin-film electrodes for neural implants," Proc. Annu. Int. Conf. IEEE Eng. Med. Biol. Soc. EMBS, pp. 5134-5137, 2012.

[13] J. M. Herrera Morales, J.-C. Souriau, and G. Simon, "Corrosion Protection of Silicon Micro Systems with Ultra-Thin Barrier Films for Miniaturized Medical Devices," ECS Trans., vol. 69, no. 26, pp. 9-26, Dec. 2015.

[14] S. F. Cogan, "Neural stimulation and recording electrodes.," Annu. Rev. Biomed. Eng., vol. 10, pp. 275309, Jan. 2008.

[15] W. Li, D. C. Rodger, E. Meng, J. D. Weiland, M. S. Humayun, and Y.-C. Tai, "Wafer-Level Parylene Packaging With Integrated RF Electronics for Wireless Retinal Prostheses," J. Microelectromechanical Syst., vol. 19, no. 4, pp. 735-742, Aug. 2010.

\section{CONTACT}

*G. K. Fedder; tel: +1-412-268-8443; fedder@,cmu.edu 